Check for updates

Cite this: RSC Adv., 2019, 9, 14692

\title{
Anion amphiphilic random copolymers and their performance as stabilizers for $\mathrm{O} / \mathrm{W}$ nanoemulsions $\uparrow$
}

\author{
Fangfang Peng, (D) Yangchuan Ke, (D)* Shichao Lu, Yi Zhao, Xu Hu \\ and Qingchun Deng
}

A series of anionic amphiphilic random copolymers with sodium $\mathrm{p}$-styrene sulfonate and dodecyl methacrylate side chains were synthesized via free radical polymerization and their properties in the formation and stabilization of nano-emulsions were investigated. Using poly(sodium p-styrene sulfonate)-ran-poly(dodecyl methacrylate) and Brij 30 as a stabilizer to prepare nanoemulsions, we obtained small droplet size and unimodal distribution nanoemulsions by a low-energy phase inversion composition (PIC) method. The p(SSS)-ran-p(LMA)-Brij 30 co-stabilized nanoemulsions show extraordinary long-term stability and heat resistance, there were almost no variations of droplet size after storing for 35 days and no phase inversion occurred when heating the temperature up to $90{ }^{\circ} \mathrm{C}$. The influence of salinity on the properties of the nanoemulsions was also discussed.

Received 23rd February 2019

Accepted 25th April 2019

DOI: 10.1039/c9ra01383k

rsc.li/rsc-advances nanoemulsion polymerizations, coatings, biotechnology, nanotechnology, medicine, pharmacology, cosmetics, and enhanced oil recovery. ${ }^{23,24}$

Due to their nanometric scale (typically in the range 20-200 $\mathrm{nm}$ ) and enhanced stability against sedimentation or creaming, nanoemulsions are in a growing number of applications. Numerous papers and reviews of nanoemulsions have been published and the fields cover emulsion polymerization, ${ }^{22}$ drug delivery, ${ }^{25}$ cosmetics, ${ }^{26}$ food industry, ${ }^{27}$ enhanced oil recovery. ${ }^{28,29}$ However, the studies of polymeric surfactants as emulsifier to stabilize nanoemulsions are very limited. Y. K. Li synthesized amphiphilic double-brush copolymers (DBCs) and used it as emulsifier to prepare nanoemulsion by highenergy ultrasonication method and obtained high stable nanoemulsions with diameter about $180 \mathrm{~nm} .{ }^{16}$ Y. S. Nam prepared $\mathrm{O} / \mathrm{W}$ nanoemulsions using an amphiphilic block copolymer, PEO- $b$-PCL as stabilizers by the phase separation of copolymers from the organic phase. In this method, a homogeneous polymer/oil mixture was dispersed in water at $80^{\circ} \mathrm{C}$ to generate embryonic emulsions, and then the emulsion size was reduced to a nanometer range through microfluidic homogenization. ${ }^{30}$ These methods require specific equipment, and not easy to implement. In contrast, low-energy methods provide an easy and scalable route to make nanoemulsions that can be achieved by a magnetic stirrer. The most widely used low energy method in literature is the phase inversion composition (PIC). Generally, when using PIC method to prepare $\mathrm{O} / \mathrm{W}$ nanoemulsions at constant temperature, water should be added to a mixture of an oil and surfactant. ${ }^{31}$ To the best of our knowledge, the report on using amphiphilic random copolymers as stabilizer to prepare nanoemulsion by low energy method hasn't been found. 
In this paper, we intend to approach a simple route for preparing $\mathrm{O} / \mathrm{W}$ nanoemulsion with anion amphiphilic random copolymers as stabilizers by PIC method. First, we synthesized a series of anionic p(SSS)-ran-p(LMA) amphiphilic random copolymers through free radical copolymerization. Then, using the $\mathrm{p}$ (SSS)-ran-p(LMA) together with nonionic Brij 30 as stabilizer, we prepared $\mathrm{O} / \mathrm{W}$ nanoemulsions by PIC method at $70{ }^{\circ} \mathrm{C} .{ }^{32}$ The droplet size, morphology, stability and interfacial tension of the nanoemulsions were evaluated. The influence of salinity on the properties of the nanoemulsions was also discussed.

\section{Experimental section}

\section{Materials}

Sodium $p$-styrenesulfonate hydrate (SSS, Aladdin Industrial Corporation, purity, 90\%), dodecyl methacrylate (LMA, Aladdin Industrial Corporation, purity, 96\%). AIBN (Xiya Chemical Industry Co. Ltd), N,N-dimethylformamide (DMF, China National Pharmaceutical Group Corporation, AR). Lauryl polyoxyethylene ether (Brij 30, Aladdin Industrial Corporation). Liquid paraffin $\left(d_{4}{ }^{20}=0.835-0.855 \mathrm{~g} \mathrm{~cm}^{-3}\right.$, cp, Tianjin Fuchen Chemical Reagents Factory). NaCl (China National Pharmaceutical Group Corporation, AR). All chemicals were used as received. Water used in this work was deionized water. Crude oil is produced in Panyu, Guangdong $\left(\rho=0.846 \mathrm{~g} \mathrm{~cm}^{-3}\right.$, and equilibrium interfacial tension of $\mathrm{O} / \mathrm{W}$ was $6.558 \mathrm{mN} \mathrm{m}^{-1}$, as seen in Fig. S1 in ESI $\dagger$ ). Water used in this work was deionized water.

\section{Synthesis}

A $250 \mathrm{~mL}$ three-necked flask was charged with sodium p-styrene sulfonate (4 g, $19.4 \mathrm{mmol}$ ), dodecyl methacrylate (2 g, 7.87 $\mathrm{mmol})$ and DMF $(80 \mathrm{~mL})$. The flask was filled with nitrogen, and AIBN $(0.24 \mathrm{~g}, 1.4 \mathrm{mmol})$ was quickly added to the reaction mixture. The flask was sealed, and backfilled with nitrogen and then allowed to reach $80{ }^{\circ} \mathrm{C}$ and was stirred for $36 \mathrm{~h}$. The reaction was stopped by exposing the solution to air and cooling to room temperature. The solvent was removed by rotary evaporators and the product was washed with ethanol before dried under vacuum.

To achieve a general understanding of the effect of hydrophilic/lipophilic ratio on the polymer properties, hydrophilic (SSS) and lipophilic (LMA) monomers were added into the reaction solution with different feed ratios as shown in Table 1.

\section{Nanoemulsion preparation}

Before emulsification, surfactants (Brij 30 and p(SSS)-ranp(LMA)) were dissolved into the oil phase under magnetic stirring. The surfactant-oil mixture and water were placed separately in a water bath at $70{ }^{\circ} \mathrm{C}$. The dosage of Brij 30 was $0.54 \mathrm{~g}$, and $\mathrm{p}$ (SSS)-ran-p(LMA) was $0.24 \mathrm{~g}$. The surfactant to oil ratio was fixed at $1: 1$. Then $20 \mathrm{~mL}$ water phase was added dropwise to the surfactant-oil (paraffin) solution in the water bath. After the emulsification, the samples were cooled at ambient temperature $\left(\sim 20^{\circ} \mathrm{C}\right)$.

\section{Characterization}

The chemical structure of the copolymers was confirmed by ${ }^{1} \mathrm{H}$ NMR analysis (AVANCE II, $400 \mathrm{MHz}$, Bruker) with $\mathrm{D}_{2} \mathrm{O}$ as a solvent. The molecular weight distributions were determined by GPC (Malvern Instruments Ltd.) equipped with a Viscotek TDA305max detector, using $\mathrm{H}_{2} \mathrm{O}$ containing $0.1 \mathrm{M} \mathrm{NaNO}_{3}$ as the eluent. The surface tension of the random copolymer aqueous solutions was measured with BZY-2 surface tensiometer

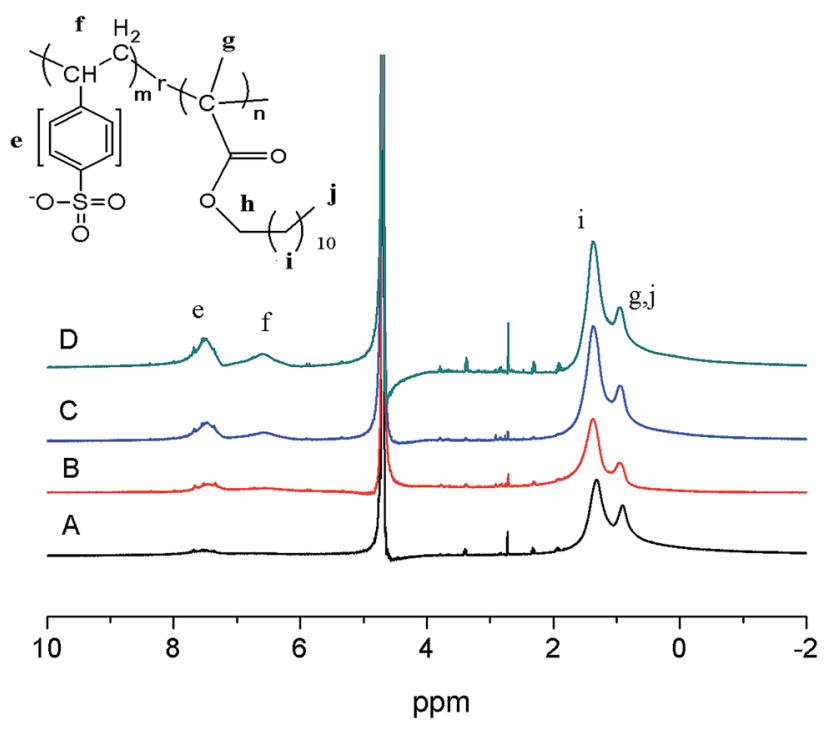

Fig. $1{ }^{1} \mathrm{H}$ NMR of $\mathrm{p}(\mathrm{SSS})$-ran-p(LMA) amphiphilic random copolymers in $\mathrm{D}_{2} \mathrm{O}$. (A) SL57, (B) SL33, (C) SL75, (D) SL42.

Table 1 Sample information of p(SSS)-ran-p(LMA) amphiphilic random copolymers ${ }^{a}$

\begin{tabular}{|c|c|c|c|c|c|c|}
\hline Samples & $\begin{array}{l}\text { Hydrophilic/lipophilic } \\
\text { [wt], feed ratio }\end{array}$ & ${ }^{b}$ Hydrophilic/lipophilic [mol], feed ratio & $M_{\mathrm{n}} \mathrm{kDa}$ & $M_{\mathrm{w}} \mathrm{kDa}$ & $M_{\mathrm{w}} / M_{\mathrm{n}}$ & Yield\% \\
\hline SL57 & $2.5 / 3.5$ & $5 / 6$ & 6168 & 7322 & 1.187 & 83.0 \\
\hline SL33 & $3.0 / 3.0$ & $7 / 6$ & 1555 & 2689 & 1.729 & 84.5 \\
\hline SL75 & $3.5 / 2.5$ & $13 / 8$ & 790 & 1376 & 1.741 & 91.0 \\
\hline SL42 & $4.0 / 2.0$ & $7 / 3$ & 636 & 928 & 1.460 & 92.3 \\
\hline
\end{tabular}

${ }^{a}$ SL-xy; (1) S represents p-styrene sulfonate, L represents dodecyl methacrylate. (2) $x y$ is the weight feed (ratio) of p-styrene sulfonate and dodecyl methacrylate, respectively. (3) Molecular weight was measured by water GPC calibrated using PEO standards. ${ }^{b}$ It's worth noting that hydrophilic/ lipophilic [wt] feed ratio was the actual amount of feed for clarity, hydrophilic/lipophilic [mol] feed ratio were calculated removing the impurities. 


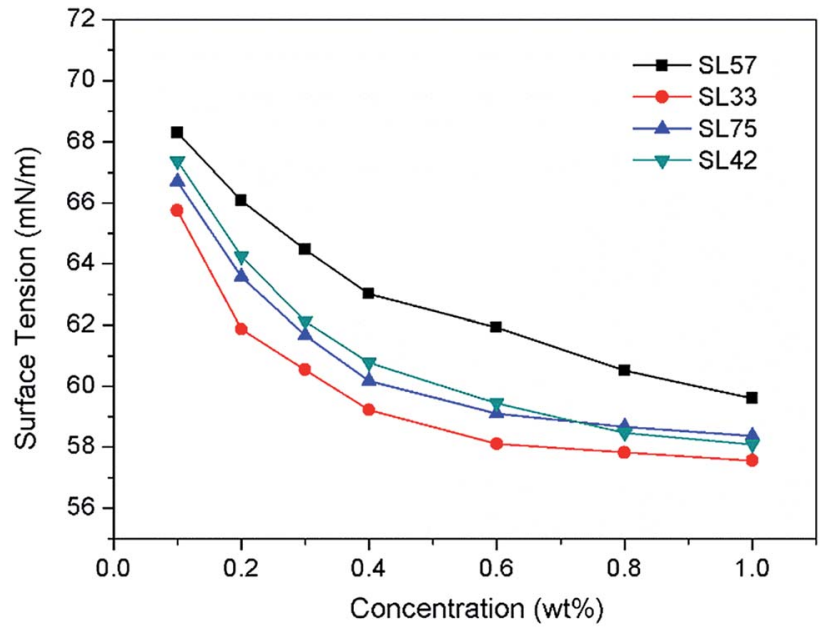

Fig. 2 Surface tension of $p(S S S)$-ran-p(LMA) solutions as a function of concentration.

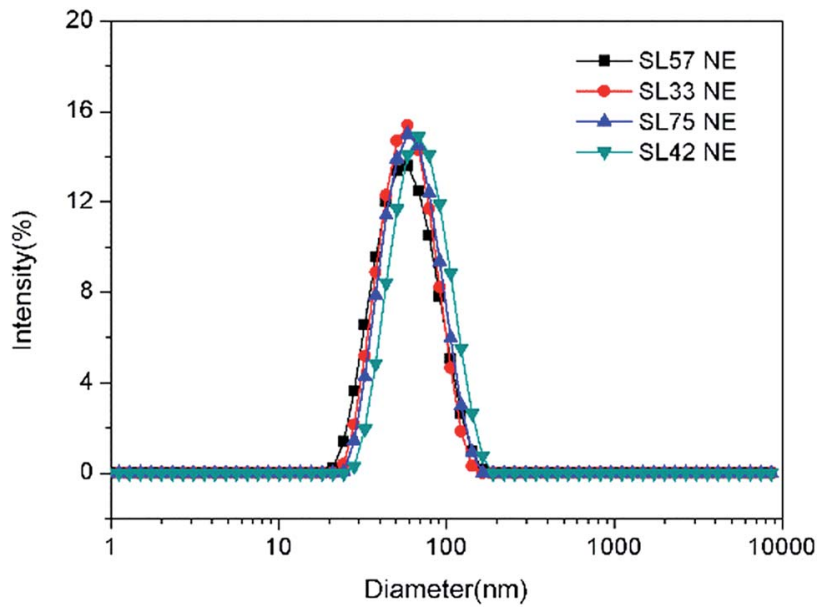

Fig. 3 Particle size and distribution of nanoemulsions measured by DLS (prepared with p(SSS)-ran-p(LMA) and Brij 30).

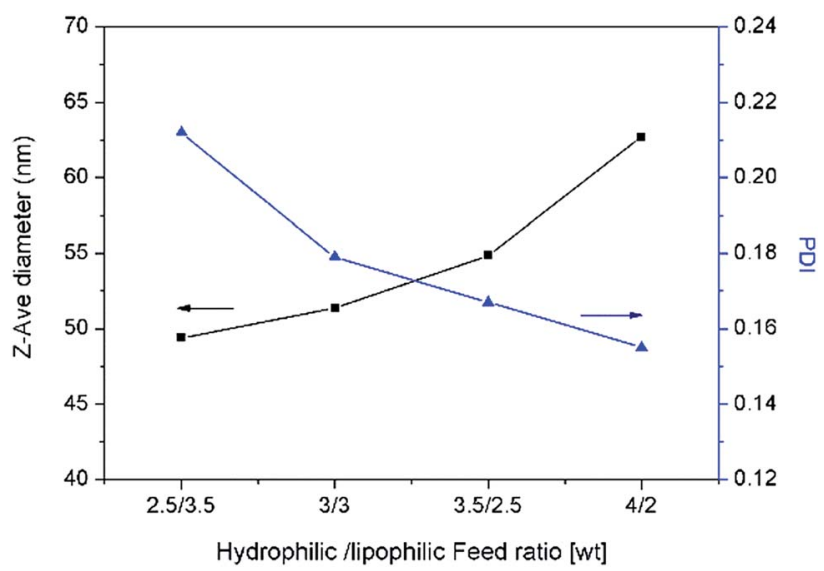

Fig. 4 Average diameter and polydispersity index(PDI) of nanoemulsion prepared with various copolymers.

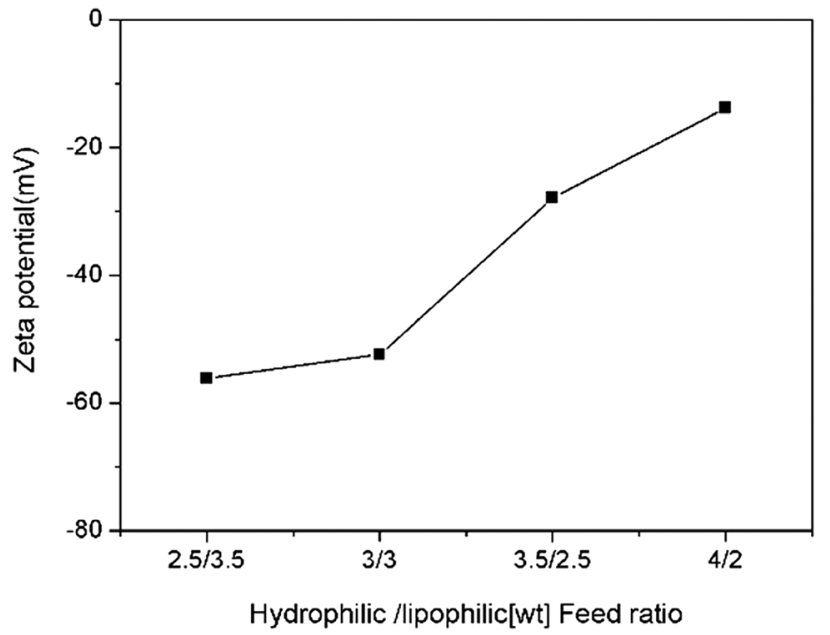

Fig. 5 Zeta potential of nanoemulsion prepared with various copolymers.

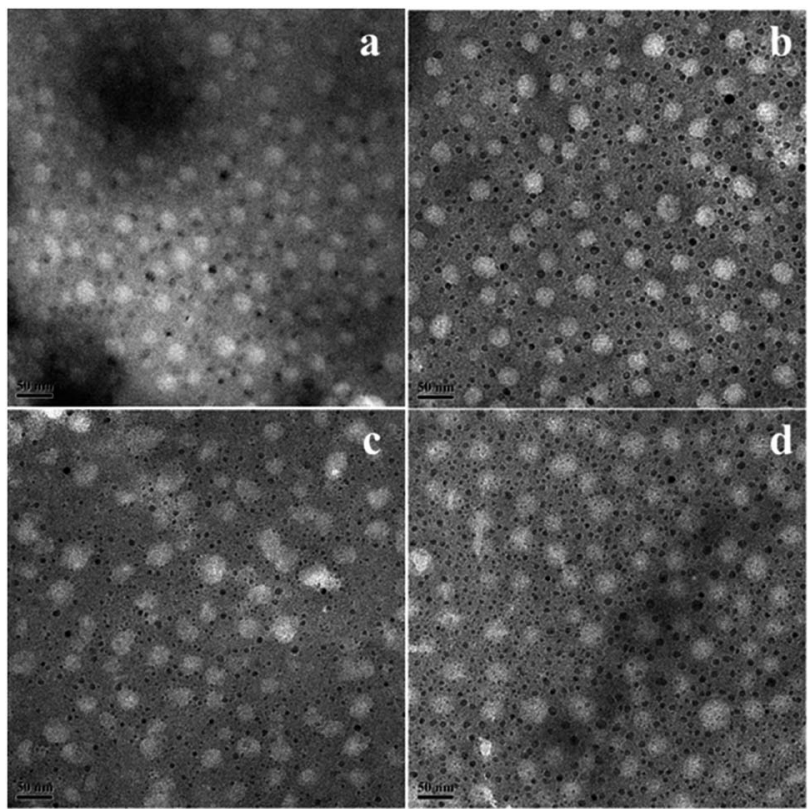

Fig. 6 TEM image of nanoemulsions (a) SL57 NE, (b) SL33 NE, (c) SL75 NE, (d) SL42 NE. The scale bar was $50 \mathrm{~nm}$.

(Shanghai Microtech Precision Instrument Co., Ltd.) via platinum plate method at $25{ }^{\circ} \mathrm{C}$. Transmission electron microscopy (TEM) measurement was taken using a Tecnai F20(FEI) transmission electron microscope. In order to facilitate the observation of the nanoemulsion droplets, $3 \mathrm{wt} \%$ phosphotungstic acid salt solution was dropped on the dried TEM samples for negative dyeing. The size and the zeta potential of nanoemulsion droplets were measured by dynamic light scattering using the Malvern Zetasizer Nano ZS, with the laser wavelength $633 \mathrm{~nm}$ and the scanning angle $173^{\circ}$ at $25 \pm 0.1{ }^{\circ} \mathrm{C}$. The zeta potential was calculated from the electrophoretic mobility by Smoluchowski's equation. The Turbiscan Lab Expert (Formulaction, France) was used to evaluate the stability of the 


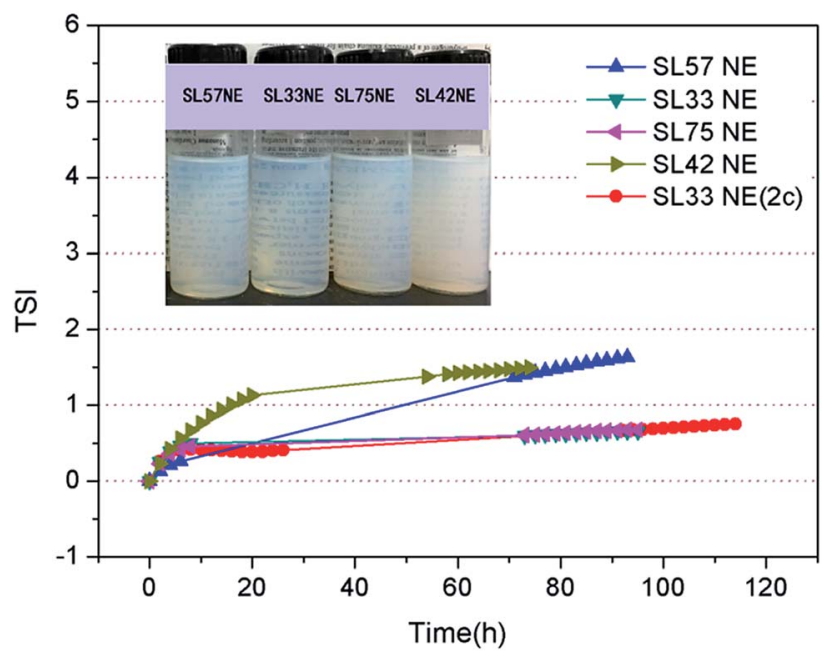

Fig. 7 TSI values of nanoemulsions stabilized by $p(S S S)$-ran-p(LMA) and Brij 30 as a function of time. (SL33 NE(2c) was the nanoemulsions same with SL33 NE except the internal phase content $(\varphi)$ is twice of the latter.) Inset photo are the appearances of nanoemulsion stored at room temperature for 7 days.

nanoemulsions at room temperature. The conductivity of the nanoemulsion was measured as a function of temperature using a Leici DDS-307A conductivity meter and a Pt/platinized electrode with a cell constant of $1.007 \mathrm{~cm}^{-1}\left(9.433 \mathrm{~cm}^{-1}\right.$ for testing nanoemulsions with $0.05 \mathrm{M} \mathrm{NaCl}$ ). Interfacial tensions of interfaces between crude oil and nanoemulsions were determined by pendant drop experiments at $45{ }^{\circ} \mathrm{C}$ using TX500C interface tensiometer (Shanghai Zhongchen Digital Technology Equipment Co., Ltd., China).

\section{Results and discussion}

\section{Polymer characterization}

The chemical composition, molecular weight and yield of the poly (sodium p-styrene sulfonate)-ran-poly(dodecyl methacrylate) copolymers are illustrated in Table $1 .{ }^{1} \mathrm{H}$ NMR spectra signals of $\mathrm{p}(\mathrm{SSS})$-ran-p(LMA) in $\mathrm{D}_{2} \mathrm{O}$ are shown in Fig. 1. $\mathrm{p}$ (SSS) $\left(-\mathrm{ph}(\mathrm{H})-\mathrm{SO}_{3} \mathrm{Na}, 7.0-8.0 \mathrm{ppm}\right.$; (H)C-ph- $\left.\mathrm{SO}_{3} \mathrm{Na}, 6.0-7.2 \mathrm{ppm}\right)$, p(dodecyl methacrylate) $\left(-\mathrm{CH}_{2}-, 1.0-1.5 \mathrm{ppm},-\mathrm{CH}_{3}, 0.6-1.0\right.$ ppm). GPC charts of the copolymers are shown in Fig. S2 (see in ESI $\dagger$ ). With an increase in the hydrophilic/lipophilic [wt] feed ratio, $M_{\mathrm{n}}$ and $M_{\mathrm{w}}$ declined dramatically, and the yield of copolymers increased.

\section{Surface tension}

The dependence of surface tension on polymer concentration in aqueous solutions is shown in Fig. 2. The decline trend of surface tension-concentration curve is similar to classic lowmolecular weight surfactants. At low concentrations, the surface tension decreases significantly with the surfactants concentration increasing, and the downward trend slows down at higher concentration. From Fig. 2 we can observe the surface tension of SL57 (with lower hydrophilic/lipophilic ratio and higher molecular weight) solution is higher than other copolymers at the same concentration, which is different with their low molecular-weight counterparts. This may be attributed to the adsorption process of polymeric surfactants at the air/water interface is influenced by molecular weight of the polymers. ${ }^{13,22}$ As we all know, the hydrophile-lipophile balance (HLB) number is relevant for the relative molecular mass of the hydrophilic/ lipophilic group in the molecule. ${ }^{33}$ While herein the polymer surfactant has a wide molecular weight distribution and

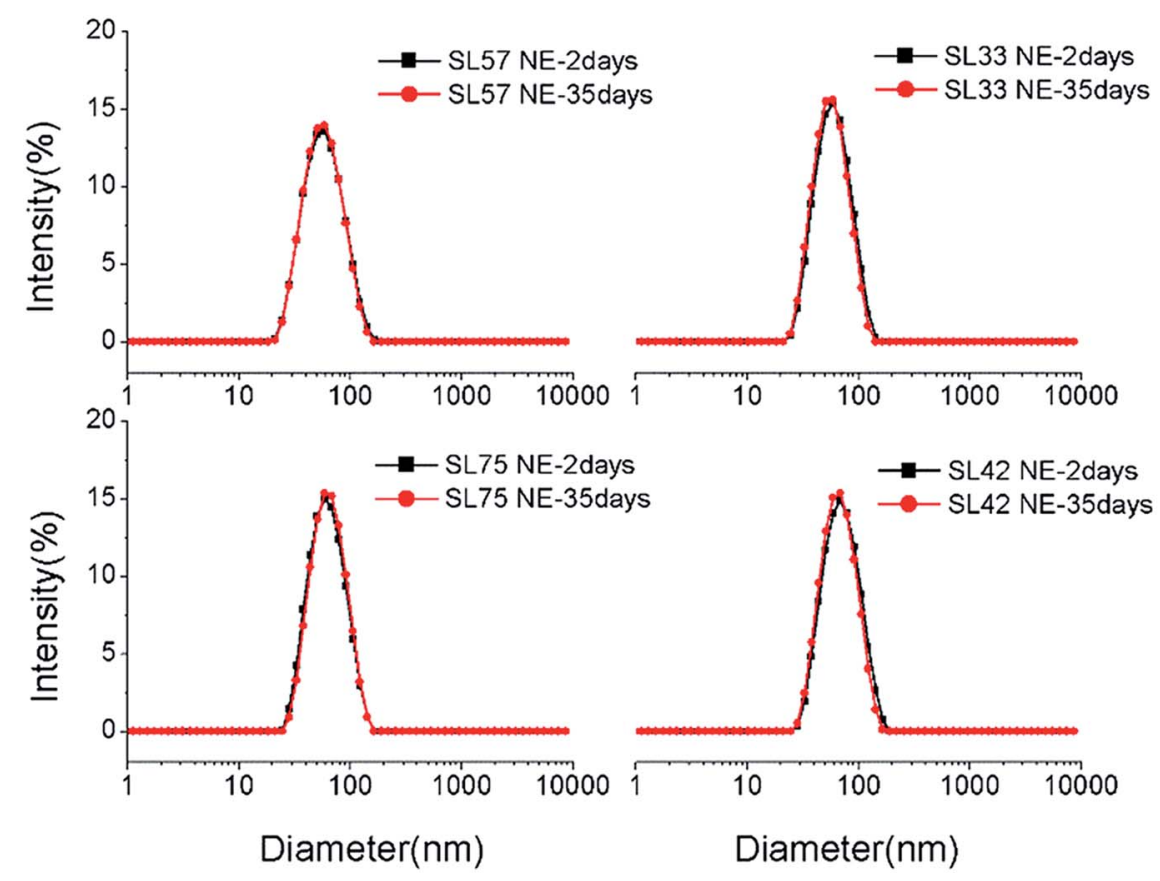

Fig. 8 Diameter variation of nanoemulsions stabilized by p(SSS)-ran-p(LMA)-Brij 30 storing for 2 and 35 days. 

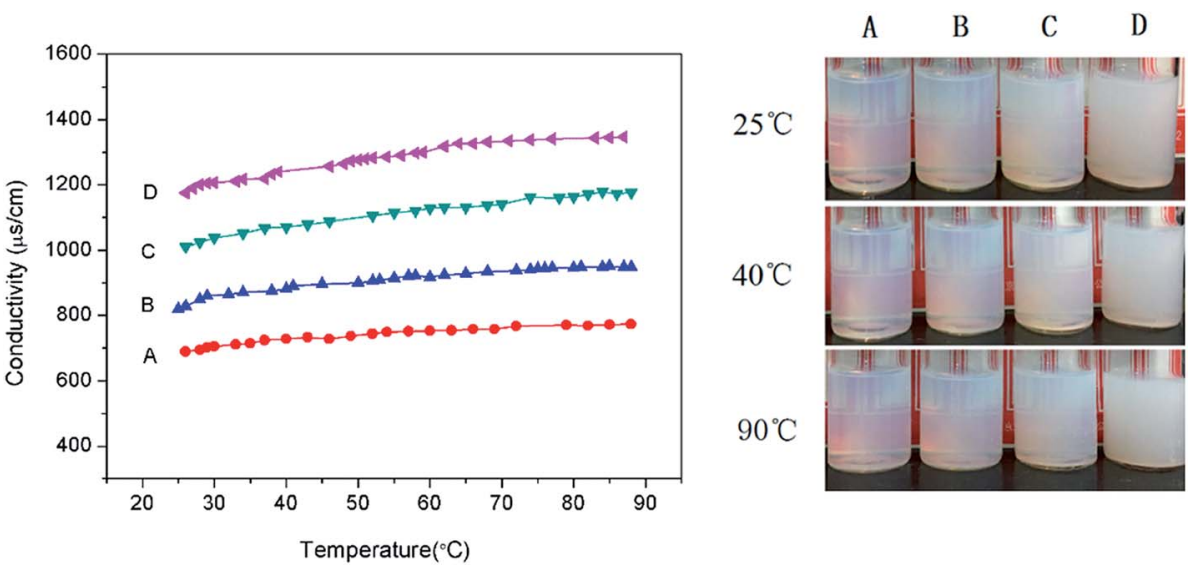

Fig. 9 Conductivity of nanoemulsions as a function of temperature. (A) SL57 NE, (B) SL33 NE, (C) SL75 NE, (D) SL42 NE (left). The photo of nanoemulsions at different temperature (right).

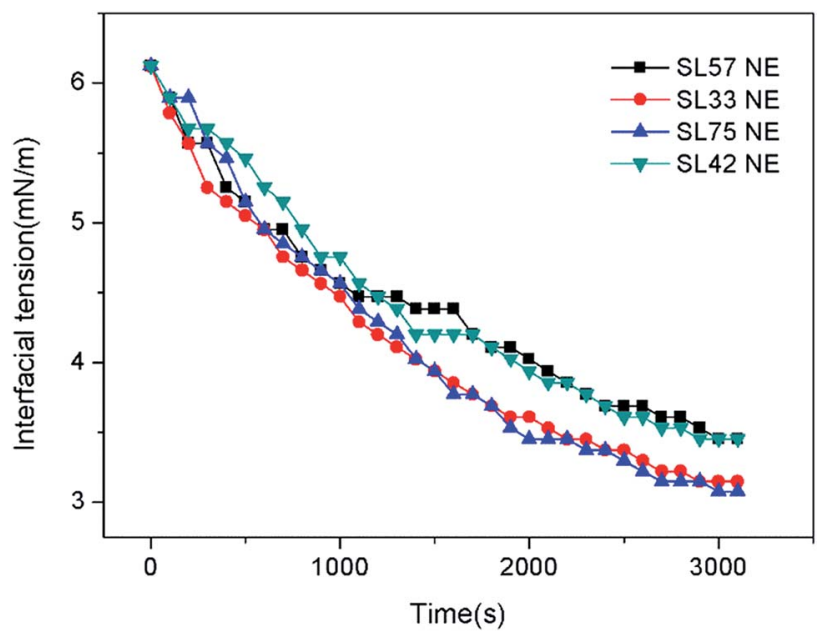

Fig. 10 Interfacial tension of crude oil/nanoemulsions as a function of time at $45^{\circ} \mathrm{C}$. fluctuation in hydrophilic/lipophilic ratios due to the free radical polymerization, thus make it inappropriate to use the hydrophilic/lipophilic ratio to estimate the HLB numbers. Moreover, it was reported that the surface activity of the polymeric surfactant can hardly be predicted by the hydrophiliclipophilic balance (HLB) due to the adsorption process of polymeric surfactants at the air/water interface is influenced by many factors, i.e., $\mathrm{pH}$, block structure and length, concentration, and topology. ${ }^{22}$

\section{Particle size and image of $\mathrm{O} / \mathrm{W}$ nanoemulsion}

The droplet size and distribution of nanoemulsions were measured by dynamic laser scattering (DLS). As shown in Fig. 3, nanoemulsion stabilized by mixed Brij 30 and p(SSS)-ran$\mathrm{p}$ (LMA) has narrow unimodal distribution. With the hydrophilic/lipophilic ratio of polymer increasing, the average (Z-Ave) diameter of the nanoemulsion droplets slightly increases from 47 to $65 \mathrm{~nm}$, and the corresponding PDI decreases from 0.21 to 0.14 , as seen in Fig. 4 . Herein due to the
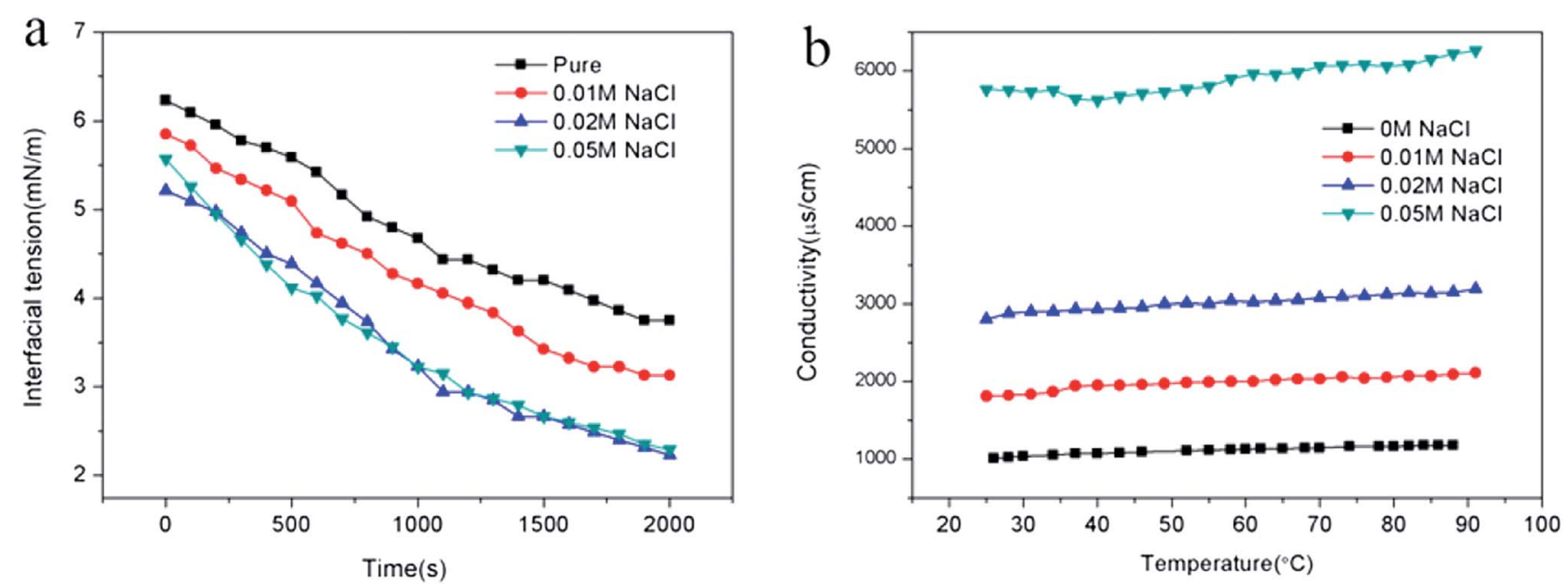

Fig. 11 Effect of salinity on interfacial tension (a) and conductivity (b) of the nanoemulsions. 
radical polymerization method was conducted, the molecular weight distribution is wide and the hydrophilic/lipophilic ratio in the polymer molecule is not fixed, thus the hydrophilic/ lipophilic ratio is a statistics data. The DLS results show that when amphiphilic random copolymer was used as a nanoemulsion stabilizer, a narrowly distributed nanoemulsion can be obtained even though the polymer surfactant has a wide molecular weight distribution and fluctuation in hydrophilic/ lipophilic ratios.

The zeta potential of the nanoemulsion as a function of hydrophilic/lipophilic ratio is shown in Fig. 5. The result shows that all the nanoemulsion samples are negatively charged. Interestingly, the value of zeta potential gradually changes from $-56.1 \mathrm{mV}$ to $-\mathbf{1 3 . 8} \mathrm{mV}$ with hydrophilic/lipophilic ratio increase, which was opposite of what we expected. This phenomenon have also been reported and discussed in previous reports. ${ }^{34-36}$ According to the literatures, the hydrophilic surfaces would lower the electrophoretic mobility, thus affect the value of zeta potentials. In this work, the surface of the nanoemulsions stabilized with higher hydrophilic/lipophilic ratio may behave more hydrophilic than of lower hydrophilic/ lipophilic ratio ones. Thus make the zeta potential value inconsistency with surface charge occurred.

The morphology of the nanoemulsion was measured by transmission electron microscopy (TEM). As seen in Fig. 6, it was consistent with the DLS result that the droplet sizes of the nanoemulsion in the TEM images were narrowly distributed and the particle size increase with hydrophilic/lipophilic ratio increasing. While discrepancy also exist, the average diameter of the nanoemulsion determined by DLS was 47 to $65 \mathrm{~nm}$, while in the TEM images, the diameter of the nanoemulsions were all smaller than DLS results, this can be explained by the hydration shell of the nanoemulsions exist during the DLS measurement. ${ }^{\mathbf{1 0}}$

It was reported that due to the formation of the nanoemulsion droplets by nucleation at a low spontaneous curvature, the bimodal size distribution is a fundamental property of the nanoemulsion when preparing nanoemulsion with PIC method. ${ }^{37}$ In this work, using our p(SSS)-ran-p(LMA)-Brij 30 costabilizers, we obtained unimodal distribution size nanoemulsions with PIC method, breaking down the argument of bimodal size distribution property of the nanoemulsion by PIC method. To figure out the reason of this phenomenon, we first catch on the reason of unimodal distribution size nanoemulsions being obtained by (sub-) PIT emulsification. When preparing nanoemulsions with (sub-) PIT method, it was proposed that due to the best geometric compromise between the volume of dispersed phase and the area that can be covered by surfactant molecules, no surfactant is wasted, thus result in the formation of unimodal distribution size nanoemulsions. ${ }^{38}$ In this present situation, it may attribute to the amphiphilic random molecules forming pseudo-Janus-type structure by dissolving the two types of side chains at the oil/water interface and could solubilize all of the oil into swollen micelles, and also no surfactant is wasted just like the (sub-) PIT method, result in unimodal distribution size nanoemulsions obtained in the PIC process.

\section{Emulsion stability}

Stability as a function of time. Emulsifying characterization of nanoemulsions stabilized by p(SSS)-ran-p(LMA)-Brij 30 was evaluated by multiple lights scattering using Turbiscan (Formulaction, France). Turbiscan has been commonly used in the petroleum, pharmaceuticals and food fields for studying stability and phase separation tendency of disperse and colloidal systems..$^{39-42}$ The nanoemulsion stability can be estimated by the variation of transmission scattering (or Back scattering) values, through which one can calculate the Turbiscan Stability Index (TSI). TSI is a parameter to estimate the stability of the nanoemulsions. A high value of the TSI indicates instability and high probability of phase separation, whereas a low TSI value indicates stability and low probability of phase separation. The index value is given by the following equation: ${ }^{39}$

$$
\mathrm{TSI}=\sum_{\mathrm{j}}\left|\operatorname{scan}_{\text {ref }}\left(h_{\mathrm{j}}\right)-\operatorname{scan}_{\mathrm{i}}\left(h_{\mathrm{j}}\right)\right|
$$

where $\mathrm{scan}_{\mathrm{ref}}$ and $\mathrm{scan}_{\mathrm{i}}$ are the initial transmission scattering value and the transmission scattering value at a given time, respectively, $h_{\mathrm{j}}$ is a given height in the measuring cell and TSI is the sum of all the scan differences in the measuring cell.

The TSI values of the nanoemulsions as a function of time are shown in Fig. 7. The result shows that the TSI of SL33 NE and SL75 NE were lower than the other SL57 NE and SL42 NE. Moreover, increase the internal phase content $\varphi\left(e_{\mathrm{x}}, \varphi(\mathrm{SL} 33 \mathrm{NE})\right.$ $=4.5 \% ; \varphi(\mathrm{SL} 33 \mathrm{NE}(2 \mathrm{c}))=9.0 \%)$, the TSI of nanoemulsion remained at a very low value. Observe the transmissionscattering value of the nanoemulsion and its variation with time, we can evaluate the transparency quantitatively and judge the stability of the nanoemulsion intuitively and qualitatively (Fig. S3 and S4, see ESI $\dagger$ ).

Diameter variation of nanoemulsions was also measured by DLS to evaluate their stability, as seen in Fig. 8, S5, and S6 in ESI. $\dagger$ Test the diameter after storing for 35 days, the result shows that the diameter and size distribution almost have no variation compering with the original data. The DLS result indicates that all the $\mathrm{p}$ (SSS)-ran-p(LMA)-Brij 30 co-stabilized nanoemulsions have an extraordinary stability. While a doubt has arisen when we compare the result of DLS with the Turbiscan result, the SL57 NE and SL42 NE seems not so stability as seen in the result of the Turbiscan method. Before explaining this phenomenon, we should stress a fact that the samples for Turbiscan measurement were the original concentrated samples, while the samples for DLS measurement were diluted about 100 times. From the TEM image (Fig. 6) of the nanoemulsions, we can see that the SL57 NE and SL42 NE respectively possess the thinnest and thickest of the hydration shell among the four nanoemulsions. The variation of transmissionscattering value with time may reflect a flocculation of the droplets, no coalescence occurred because of the strong repulsive interactions (such as steric repulsion) among the droplets when place for 35 days. When the nanoemulsions were diluted, the droplets disperse again.

Conductivity of the nanoemulsions. The conductivity of the nanoemulsions as a function of temperature was measured 
under constant stirring, as shown in Fig. 9. The nanoemulsions are insensitivity to temperature at the range of $25-90{ }^{\circ} \mathrm{C}$ (To prevent water evaporation, the conductivity of nanoemulsions above $90{ }^{\circ} \mathrm{C}$ was not measured). This result was verified by the appearances of nanoemulsions (see the right photo). Even heated up to $90{ }^{\circ} \mathrm{C}$, there was no phase inversion observed. The insensitivity to temperature nanoemulsions has also been studied in previous reports. ${ }^{\mathbf{4 3 , 4 4}}$ It was adjudged that with temperature raise, the nonionic surfactants become more lipophilic, while the ionic surfactants become more hydrophilic, and the opposite temperature effects of the two types of surfactants cancel out. It should be pointed out that the test temperature range of conductivity is actually about $15-90{ }^{\circ} \mathrm{C}$. Between $15-25{ }^{\circ} \mathrm{C}$, the conductivity of all the nano-emulsions have experienced a slightly decline at the start of the measurement, which is not shown in this paper. The reason for this phenomenon needs further investigation.

Interfacial tension of nanoemulsions. Interfacial tension is a very important property of emulsion in some application such as enhanced oil recovery. The dynamic interfacial tension of crude oil/nanoemulsions was measured by pendant drop experiments at $45{ }^{\circ} \mathrm{C}$ (Fig. 10). The equilibrium interfacial tension of crude oil/water was $6.558 \mathrm{mN} \mathrm{m}^{-1}$, as seen in Fig. S1. $\dagger$ The four nanoemulsions can divide into two groups according to their interfacial tension of $3100 \mathrm{~s}$ of pendant drop formation. The one group was SL75NE and SL33NE, their interfacial tension are close to each other and the value was approximately $3.1 \mathrm{mN} \mathrm{m}^{-1}$. The other group was SL57NE and SL42NE and their interfacial tension was approximately $3.5 \mathrm{mN}$ $\mathrm{m}^{-1}$.

Effect of salinity on nanoemulsion. To determine the effect of salinity on nanoemulsions, four different $\mathrm{NaCl}$ concentration solutions $(0,0.01,0.02$, and $0.05 \mathrm{M})$ were used to prepare nanoemulsions, as shown in Fig. 11. From Fig. 11a, we can see that the interfacial tension of oil/nanoemulsions reduced gradually after adding $\mathrm{NaCl}$ from 0 to $0.02 \mathrm{M}$. When adding $\mathrm{NaCl}$ concentration up to $0.05 \mathrm{~mol} \mathrm{~L}^{-1}$, the interfacial tension of nanoemulsions showed no more reduction comparing with nanoemulsions of $0.02 \mathrm{M} \mathrm{NaCl}$. We deduce that the decrease of interfacial tension is due to the reduction of the kinetic repulsive adsorption barrier between like-charged emulsifiers and oil droplets by salt addition. ${ }^{45}$ Fig. $11 \mathrm{~b}$ presents the curves of the conductivity vary with temperature of nanoemulsions performed with different $\mathrm{NaCl}$ concentration solutions. Even heated up to $90{ }^{\circ} \mathrm{C}$, there have no phase inversion occurred. Indicating a good heat resistance was maintained when the salinity of $\mathrm{NaCl}$ was up to $0.05 \mathrm{~mol} \mathrm{~L}^{-1}$.

\section{Conclusions}

By adjusting the hydrophilic/lipophilic ratios, a series of anionic p(SSS)-ran-p(LMA) amphiphilic random copolymers were synthesized through free radical copolymerization. Using the p(SSS)-ran-p(LMA) together with nonionic Brij 30 as stabilizer, small droplet sizes and unimodal distribution nanoemulsions were prepared by phase inversion composition (PIC) method. The stability of the anion-nonionic mixed surfactant stabilized nanoemulsions was evaluated by multiple light scattering and dynamic light scattering. Results show that the p(SSS)-ranp(LMA)-Brij 30 co-stabilized nanoemulsions show extraordinary long-term stability and heat resistant, there were almost no variations of droplet size after storing for 35 days and no phase inversion occurred when heating up to $90{ }^{\circ} \mathrm{C}$. Adding a small amount of salt (i.e. less than $0.05 \mathrm{M} \mathrm{NaCl}$ ) will reduce the interfacial tension and have not significant influence on their heat resistance property. Because of narrow droplet size distribution and excellent stability properties of their stabilized nanoemulsions as well as they are easy to be synthesized, the amphiphilic random copolymers have significant potential applications in the industrial areas such as nanoemulsion polymerizations, coatings, nanotechnology, and enhanced oil recovery.

\section{Conflicts of interest}

There are no conflicts to declare.

\section{Acknowledgements}

This work was financially supported by the National Natural Science Foundation of China (Grant No. 51674270), National Major Project (Grant No. 2017ZX05009-003), Major project of the National Natural Science Foundation of China (No. 51490650), and the Foundation for Innovative Research Groups of the National Natural Science Foundation of China (Grant No. 51821092 and 51521063).

\section{References}

1 G. Xie, P. Krys, R. D. Tilton and K. Matyjaszewski, Heterografted Molecular Brushes as Stabilizers for Waterin-Oil Emulsions, Macromolecules, 2017, 50(7), 2942-2950.

2 Y. Xia, B. D. Olsen and J. A. Kornfield, Efficient Synthesis of Narrowly Dispersed Brush Copolymers and Study of Their Assemblies: The Importance of Side Chain Arrangement, $J$. Am. Chem. Soc., 2009, 131(51), 18525-18532.

3 H. Zhu, G. Deng and Y. Chen, Amphiphilic polymer brushes with alternating PCL and PEO grafts through radical copolymerization of styrenic and maleimidic macromonomers, Polymer, 2008, 49(2), 405-411.

4 X. Liu, C. Yi, Y. Zhu, Y. Yang, J. Jiang, Z. Cui and M. Jiang, Pickering emulsions stabilized by self-assembled colloidal particles of copolymers of P(St-alt-MAn)-co-P(VM-alt-MAn), J. Colloid Interface Sci., 2010, 351(2), 315-322.

5 T. Kawata, A. Hashidzume and T. Sato, Micellar Structure of Amphiphilic Statistical Copolymers Bearing Dodecyl Hydrophobes in Aqueous Media, Macromolecules, 2007, 40(4), 1174-1180.

6 Y. Hirai, T. Terashima, M. Takenaka and M. Sawamoto, Precision Self-Assembly of Amphiphilic Random Copolymers into Uniform and Self-Sorting Nanocompartments in Water, Macromolecules, 2016, 49(14), 5084-5091. 
7 M. Matsumoto, T. Terashima, K. Matsumoto, M. Takenaka and M. Sawamoto, Compartmentalization Technologies via Self-Assembly and Cross-Linking of Amphiphilic Random Block Copolymers in Water, J. Am. Chem. Soc., 2017, 139(21), 7164-7167.

8 L. Gu, Z. Shen, S. Zhang and G. L. Lu, Novel Amphiphilic Centipede-Like Copolymer Bearing Polyacrylate Backbone and Poly(ethylene glycol) and Polystyrene Side Chains, Macromolecules, 2007, 40(13), 4486-4493.

9 T. Stephan, S. Muth and M. Schmidt, Shape Changes of Statistical Copolymacromonomers: From Wormlike Cylinders to Horseshoe-and Meanderlike Structures, Macromolecules, 2002, 35, 9857-9860.

10 Y. Chenglin, Y. Yiqun, Z. Ye, L. Na, L. Xiaoya, L. Jing and J. Ming, Self-assembly and emulsification of poly\{[styrenealt-maleic acid]-co-[styrene-alt-(N-3,4-dihydroxyphenylethylmaleamic acid)]\}, Langmuir, 2012, 28(25), 9211-9222.

11 T. J. Neal, D. L. Beattie, S. J. Byard, G. N. Smith, M. W. Murray, N. S. J. Williams, S. N. Emmett, S. P. Armes, S. G. Spain and O. O. Mykhaylyk, Self-Assembly of Amphiphilic Statistical Copolymers and Their Aqueous Rheological Properties, Macromolecules, 2018, 51(4), 14741487.

12 H. Matsuoka, M. Hachisuka, K. Uda, T. Onishi and S. Ozoe, Why Ionic Amphiphilic "Block" Copolymer Can Be Nonsurface Active? Comparison of Homopolymer, Block and Random Copolymers of Poly(styrenesulfonate), Chem. Lett., 2012, 41(10), 1063-1065.

$13 \mathrm{H}$. Matsuoka, H. Chen and K. Matsumoto, Molecular weight dependence of non-surface activity for ionic amphiphilic diblock copolymers, Soft Matter, 2012, 8(35), 9140.

14 P. Kaewsaiha, K. Matsumoto and H. Matsuoka, Non-Surface Activity and Micellization of Ionic Amphiphilic Diblock Copolymers in Water. Hydrophobic Chain Length Dependence and Salt Effect on Surface Activity and the Critical Micelle Concentration, Langmuir, 2005, 21(22), 9938-9945.

15 P. Fontaine, J. Daillant and P. Guenoun, Spontaneous Buckling Induced by the Adsorption of Charged Copolymers at the Air-Water Interface, J. Phys. II, 1997, $7(3), 401-407$.

16 Y. Li, J. Zou, B. P. Das, M. Tsianou and C. Cheng, WellDefined Amphiphilic Double-Brush Copolymers and Their Performance as Emulsion Surfactants, Macromolecules, 2012, 45(11), 4623-4629.

17 T. Futamura and M. Kawaguchi, Characterization of paraffin oil emulsions stabilized by hydroxypropyl methylcellulose, $J$. Colloid Interface Sci., 2012, 367(1), 55-60.

18 D. Exerowa, G. Gotchev and T. Kolarov, Interaction Forces in Thin Liquid Films Stabilized by Hydrophobically Modified Inulin Polymeric Surfactant. 2 Emulsion Films, Langmuir, 2007, 23(4), 1711-1715.

19 T. F. Tadros, A. Vandamme, B. Levecke, K. Booten and C. V. Stevens, Stabilization of emulsions using polymeric surfactants based on inulin, Adv. Colloid Interface Sci., 2004, 108-109, 207-226.
20 J. P. Magnusson, A. Khan and G. A. Pasparakis, Ion-Sensitive "Isothermal" Responsive Polymers Prepared in Water, J. Am. Chem. Soc., 2008, 130(33), 10852-10853.

21 D. Wang, T. Wu and X. J. Wan, Purely Salt-Responsive Micelle Formation and Inversion Based on a Novel Schizophrenic Sulfobetaine Block Copolymer: Structure and Kinetics of Micellization, Langmuir, 2007, 23(23), 11866-11874.

22 P. Raffa, D. A. Wever, F. Picchioni and A. A. Broekhuis, Polymeric Surfactants: Synthesis, Properties, and Links to Applications, Chem. Rev., 2015, 115(16), 8504-8563.

23 T. Tadros, Polymeric surfactants in disperse systems, Adv. Colloid Interface Sci., 2009, 147-148, 281-299.

24 A. Laschewsky, U. C. Louvain, D. Chimie and P. L. Pasteur, Molecular Concepts, Self-Organisation and Properties of Polysoaps, Advances in Polymer Science, Springer-Verlag Berlin Heidelberg, 1995, vol. 124, pp. 1-86.

25 Y. Singh, J. G. Meher, K. Raval, F. A. Khan, M. i Chaurasia, N. K. Jain and M. K. Chourasia, Nanoemulsion: Concepts, development and applications in drug delivery, $J$. Controlled Release, 2017, 252, 28-49.

26 M. N. Yukuyama, D. D. M. Ghisleni, T. J. A. Pinto and N. A. Bou-Chacra, Nanoemulsion: process selection and application in cosmetics - a review, Int. J. Cosmet. Sci., 2016, 38, 13-24.

27 A. Gupta, H. B. Eral, T. A. Hatton and P. S. Doyle, Nanoemulsions: formation, properties and applications, Soft Matter, 2016, 12, 2826-2841.

28 N. Kumar and A. Mandal, Thermodynamic and physicochemical properties evaluation for formation and characterization of oil-in-water nanoemulsion, J. Mol. Liq., 2018, 266, 147-159.

29 N. Kumar and A. Mandal, Surfactant Stabilized Oil-in-Water Nanoemulsion: Stability, Interfacial Tension, and Rheology Study for Enhanced Oil Recovery Application, Energy Fuels, 2018, 32, 6452-6466.

30 Y. S. Nam, J. W. Kim, J. Shim, S. H. Han and H. K. Kim, Nanosized emulsions stabilized by semisolid polymer interphase, Langmuir, 2010, 26(16), 13038-13043.

31 A. Gupta, A. Z. M. Badruddoza and P. S. Doyle, A General Route for Nanoemulsion Synthesis Using Low-Energy Methods at Constant Temperature, Langmuir, 2017, 33(28), 7118-7123.

32 L. Yu, C. Li, J. Xu, J. Hao and D. Sun, Highly stable concentrated nanoemulsions by the phase inversion composition method at elevated temperature, Langmuir, 2012, 28(41), 14547-14552.

33 I. J. Lin, J. P. Friend and Y. Zimmels, The effect of structural modifications on the hydro-philic-lipophilic banlance of ionic surfactants, J. Colloid Interface Sci., 1973, 45(02), 378386.

34 O. El-Gholabzouri, M. A. Cabrerizo and R. Hidalgo-A lvarez, The Surface Charge Density Influence on the Electrokinetic Properties of Model Colloids: Solvent Composition Effect, J. Colloid Interface Sci., 1999, 214, 243-250. 
35 L. Vorwerg, M. Antonietti and K. Tauer, Electrophoretic mobility of latex particles: effect of particle size and surface structure, Colloids Surf., A, 1999, 150, 129-135.

36 B. R. Midmore and R. J. Hunter, The Effect of Electrolyte Concentration and Co-ion Type on the $\zeta$-Potential of Polystyrene Latices, J. Colloid Interface Sci., 1988, 122(2), 521-529.

37 K. Roger, B. Cabane and U. Olsson, Emulsification through surfactant hydration: the PIC process revisited, Langmuir, 2011, 27(2), 604-611.

38 K. Roger, B. Cabane and U. Olsson, Formation of 10-100 nm size-controlled emulsions through a sub-PIT cycle, Langmuir, 2010, 26(6), 3860-3867.

39 J. Santos, N. Calero, L. A. Trujillo-Cayado, M. C. Garcia and J. Munoz, Assessing differences between Ostwald ripening and coalescence by rheology, laser diffraction and multiple light scattering, Colloids Surf., B, 2017, 159, 405-411.

40 A. L. Marquez, A. Medrano, L. A. Panizzolo and J. R. Wagner, Effect of calcium salts and surfactant concentration on the stability of water-in-oil (w/o) emulsions prepared with polyglycerol polyricinoleate, J. Colloid Interface Sci., 2010, 341(1), 101-108.

41 D. D. Kaombe, M. Lenes, K. Toven and W. R. Glomm, Turbiscan as a Tool for Studying the Phase Separation Tendency of Pyrolysis Oil, Energy Fuels, 2013, 27(3), 14461452.

42 C. Carbone, T. Musumeci, M. R. Lauro and G. Puglisi, Ecofriendly aqueous core surface-modified nanocapsules, Colloids Surf., B, 2015, 125, 190-196.

43 R. Antón, H. Rivas and J.-L. Salager, Surfactant-Oil-Water Systems near the Affinity Inversion. Part X: Emulsions Made with Anionic-Nonionic Surfactant Mixtures, J. Dispersion Sci. Technol., 1996, 17(6), 553-566.

44 Z. Mei, S. Liu, L. Wang, J. Jiang, J. Xu and D. Sun, Preparation of positively charged oil/water nano-emulsions with a subPIT method, J. Colloid Interface Sci., 2011, 361(2), 565-572.

45 J. N. Dong, A. J. Worthen, L. M. Foster, Y. S. Chen and K. P. Johnston, Modified Montmorillonite Clay Microparticles for Stable Oil-in Seawater Emulsions, ACS Appl. Mater. Interfaces, 2014, 6, 11502-11513. 